\title{
Dynamical properties of liquid Al near melting: An orbital-free molecular dynamics study
}

\author{
D. J. González,* L. E. González, and J. M. López \\ Departamento de Física Teórica, Universidad de Valladolid, Valladolid, Spain \\ M. J. Stott \\ Department of Physics, Queen's University, Kingston, Ontario, Canada
}

(Received 9 November 2001; published 8 April 2002)

\begin{abstract}
The static and dynamic structure of liquid $\mathrm{Al}$ is studied using the orbital free ab initio molecular dynamics method. Two thermodynamic states along the coexistence line are considered, namely $\mathrm{T}=943$ and $1323 \mathrm{~K}$, for which $\mathrm{x}$-ray and neutron scattering data are available. A kinetic-energy functional which fulfills a number of physically relevant conditions is employed, along with a local first-principles pseudopotential. In addition to a comparison with experiment, we also compare our $a b$ initio results with those obtained from conventional molecular-dynamics simulations using effective interionic pair potentials derived from second-order pseudopotential perturbation theory.
\end{abstract}

DOI: 10.1103/PhysRevB.65.184201

PACS number(s): 71.15.Pd, 61.20.Lc, 71.15.Mb, 61.25.Mv

\section{INTRODUCTION}

Molecular dynamics (MD) methods have a long tradition as a useful technique to study the properties of liquid systems, and the last 15 years have witnessed a large spread in the application of $a b$ initio molecular-dynamics methods, based on density-functional theory. This theory allows the calculation of the ground-state electronic energy of a collection of atoms for given nuclear positions, ${ }^{1,2}$ and also yields the forces on the nuclei via the Hellmann-Feynman theorem. It enables one to perform molecular-dynamics simulations where the nuclear positions evolve according to classical mechanics, whereas the electronic subsystem follows adiabatically.

In this paper we present the results of an ab initio molecular-dynamics simulation of the static and dynamic properties of liquid $\mathrm{Al}$ at thermodynamic conditions around the triple point. Liquid aluminum has usually been considered as a simple metal in which the core electrons forming the ion can be clearly distinguished from the valence electrons; moreover, the core electrons do not significantly overlap with those of neighboring ions. Therefore, the system consists of a binary mixture of ions and valence electrons, where the former may be treated classically whereas the electrons must be treated quantum mechanically.

However, in wide regions of the density-temperature plane, simple metals have usually been treated as an effective one-component fluid of ions interacting by means of densitydependent effective interionic pair potentials, derived from ionic pseudopotentials by applying second-order perturbation theory. This approach, which will be referred to as the linearresponse theory (LRT), has often been used as the starting point for the study of the static and dynamic properties of the simple metals. ${ }^{3-6}$ It has also been the approach followed in most studies on the static structure of liquid aluminum. ${ }^{7-11}$ Among them, we mention the work of Dagens et al. ${ }^{8}$ who obtained an effective interionic pair potential, derived from a nonlocal pseudopotential which was constructed from the valence charge density induced by an $\mathrm{Al}^{+3}$ ion placed in an electron gas at the metallic density. From this potential,
Jacucci et al. ${ }^{9}$ calculated the static structure factor of liquid aluminum by means of MD simulations; their results showed fair agreement with experiment, with a main peak somewhat higher. Also, Hafner and Jank ${ }^{11}$ studied the liquid static structure of aluminum by means of an effective interionic pair potential derived from an $a b$ initio pseudopotential originally developed by Harrison, ${ }^{12}$ whereas the corresponding liquid static structure was derived by means of MD simulations. In their calculation of the pseudopotential, the authors used the coefficient for the exchange-correlation potential between the core and valence electrons as a fitting parameter in order to obtain agreement with the experimental static structure factor.

Whereas the previous work dealt only with the static properties of liquid $\mathrm{Al}$ near melting, the work of Ebbsjo et l. $^{7}$ also considered some dynamic properties. In fact, these authors performed MD simulations for three different interionic pair potentials, two of them based on nonlocal pseudopotentials and the other one based on the local Ashcroft's pseudopotential, ${ }^{13}$ which showed a rather different shape, especially outside the repulsive core. Despite these differences, all approaches gave fairly similar results for the liquid static structure which agreed well with the experimental data, whereas the main discrepancies appeared in the dynamic structure.

A rather different approach was followed by Chihara and co-workers. ${ }^{14,15}$ Their quantum-hypernetted-chain (QHNC) method treats ions and electrons on a basically equal footing by combining liquid-state integral equations with the density-functional formalism. Moreover, it does not rely on the pseudopotential ideas, gives rise to a self-consistent scheme to determine the liquid static structure, and yields an effective interionic pair potential which depends on the particular liquid static structure.

Although the LRT approach has produced reasonable results for liquid alkali metals, when the valence of the system is increased, its validity becomes more questionable. In addition, even for alkali metals the LRT is less justifiable for thermodynamic states approaching the critical point, and it is certainly wrong near the critical point. This limitation of the 
"standard" theory estimulated the use of first-principles molecular-dynamics techniques, ${ }^{16-20}$ where the electronic density, total energy and forces are obtained by using the Kohn-Sham (KS) formulation of the density functional theory (DFT). ${ }^{2}$ However, the computational demands of these $a b$ initio methods, where KS orbitals are used to describe the electronic density and to compute the electronic kinetic energy exactly, grow very rapidly with the system size, and their memory requirement is also quite large. These considerations have restricted the sizes of the systems studied so far to about 60 atoms, and have limited simulation times to around $2-5 \mathrm{ps}$ in the cases of $\mathrm{Rb}, \mathrm{Cs}$, and $\mathrm{Hg},{ }^{16-19}$ and $64-128$ atoms, with simulation times of $0.15-0.85 \mathrm{ps}$ in the case of $\mathrm{Na}^{20}$ These limitations can be at least partly overcome if the exact calculation of the electronic kinetic energy is given up in favor of an approximate kinetic energy functional of the electronic density. Within this scheme ${ }^{21}$ the number of variables describing the electronic state are enormously reduced, especially for large systems, enabling the study of larger systems for longer simulation times. This approach was already used for several studies on solids, ${ }^{22}$ clusters, ${ }^{23}$ and some liquid metals ( $\mathrm{Li}, \mathrm{Na}, \mathrm{Mg}$, and $\mathrm{Al}$ ) near melting. ${ }^{24,26}$ We recently presented ${ }^{27}$ an application of this method to study the static structure and some dynamic properties of expanded liquid Cs, for which experimental data is available. ${ }^{28}$ In that study 125 particles were used, and the simulation time was $17-35$ ps after an equilibration time of $11-25$ ps. Also, another study ${ }^{25}$ with 205 particles for liquid Al gave results for the static structure in good agreement with experiment. Recently, Anta et al. ${ }^{26}$ also applied the same scheme to study the ionic and electronic static structure of liquid Al near melting, leading to results for the static structure factor in excellent agreement with experiment.

The static structure factor of liquid $\mathrm{Al}$ has been measured by both neutron ${ }^{29,30}$ and $\mathrm{x}$-ray ${ }^{31,32}$ diffraction. The dynamical structure of liquid $\mathrm{Al}$ near the triple point was also investigated recently by Scopigno et al. $^{33}$ using inelastic X-ray scattering (IXS). Note that the high value of the adiabatic sound speed for liquid $\mathrm{Al}(\approx 4800 \mathrm{~m} / \mathrm{s})$ prevents the use of the inelastic neutron-scattering technique for investigating the collective excitations for small- $q$ values (roughly, for $q$ $\leqslant q_{p}$, with $q_{p} \approx 2.70 \AA^{-1}$ being the main peak position of the static structure factor). Those IXS experiments investigated the wave-vector region $0.05 q_{p} \leqslant q \leqslant 0.5 q_{p}$, obtaining several dynamical features previously observed in the liquid alkali metals, such as the existence of collective excitations up to $q$ values larger that $0.5 q_{p}$, which exhibit a positive dispersion in the sound velocity with respect to the hydrodynamic value.

The layout of the paper is as follows. In Sec. II we briefly describe the theory used in the orbital-free $a b$ initio molecular-dynamics (OF-AIMD) simulations, giving some technical details, and focusing on the two problematic issues, namely, the kinetic-energy functional and the local pseudopotentials needed to characterize the ion-electron interaction. In Sec. III we present and discuss the results of the ab initio simulations; moreover, they are compared with further classical molecular dynamics (CMD) simulations that we have performed based on LRT and the QHNC potentials, and with the available experimental data. Finally some conclusions are drawn and possible ideas for further improvements are suggested.

\section{THEORY}

The total potential energy of a system of $N$ classical ions enclosed in a volume $V$, and interacting with $N_{\mathrm{e}}=N Z$ valence electrons through a local electron-ion potential $v(r)$, is written, within the Born-Oppenheimer approximation, as the sum of the direct ion-ion Coulombic interaction energy, and the ground-state energy of the electronic system subject to the external potential created by the ions, $V_{\text {ext }}\left(\vec{r},\left\{\vec{R}_{l}\right\}\right)$ $=\sum_{i=1}^{N} v\left(\left|\vec{r}-\vec{R}_{i}\right|\right)$,

$$
E\left(\left\{\vec{R}_{l}\right\}\right)=\sum_{i<j} \frac{Z^{2}}{\left|\vec{R}_{i}-\vec{R}_{j}\right|}+E_{g}\left[\rho_{g}(\vec{r}), V_{\mathrm{ext}}\left(\vec{r},\left\{\vec{R}_{l}\right\}\right)\right],
$$

where $\rho_{g}(\vec{r})$ is the ground-state electronic density and $\vec{R}_{l}$ are the ionic positions. According to LRT, the ground-state electronic density is given, in reciprocal space, by

$$
\begin{gathered}
\rho_{g}^{\mathrm{LRT}}(\vec{q})=\left(\sum_{j} e^{i \vec{q} \vec{R}_{j}}\right) n^{\mathrm{LRT}}(q) \equiv F(\vec{q}) n^{\mathrm{LRT}}(q) \\
n^{\mathrm{LRT}}(q)=\chi\left(q, \rho_{0}\right) v(q),
\end{gathered}
$$

where $\chi\left(q, \rho_{0}\right)$ is the response function of a uniform electron gas of density $\rho_{0}=N Z / V$. Accordingly, the ground-state electronic density is a superposition of spherically symmetric pseudoatomic densities around each ion, i.e.,

$$
\rho_{g}^{\mathrm{LRT}}(\vec{r})=\sum_{j} n^{\mathrm{LRT}}\left(\left|\vec{r}-\vec{R}_{j}\right|\right)
$$

and the electronic ground-state energy is

$$
\begin{gathered}
E_{g}^{\mathrm{LRT}}=E_{v}\left[\rho_{0}\right]+\sum_{i<j} \phi_{\text {ind }}\left(R_{i j}\right) \\
\phi_{\text {ind }}(q)=\chi\left(q, \rho_{0}\right) v^{2}(q),
\end{gathered}
$$

where $E_{v}\left[\rho_{0}\right]$ is a structure-independent term. Within the LRT, the total potential energy can be written as a sum of a structure-independent term and a sum over pairs of an effective interionic pair potential $\phi_{\text {eff }}(R)=Z^{2} / R+\phi_{\text {ind }}(R)$.

Alternatively, DFT shows that the ground-state electronic density can be obtained by minimizing the energy functional $E[\rho]$, and the minimum value of the functional gives the ground-state energy of the electronic system. The energy functional can be written

$$
E_{g}[\rho(\vec{r})]=T_{s}[\rho]+E_{\mathrm{ext}}[\rho]+E_{H}[\rho]+E_{\mathrm{xc}}[\rho]
$$

where the terms represent, respectively, the electronic kinetic energy $T_{s}[\rho]$ of a non-interacting system of density $\rho(\vec{r})$, the energy of interaction with the external potential due to the ions, 
TABLE I. Thermodynamic states studied in this work, along with some simulation details.

\begin{tabular}{lcccc}
\hline \hline & $T(\mathrm{~K})$ & $\mathrm{N}$ & $\rho\left(\AA^{-3}\right)$ & $E_{\text {Cut }}(\mathrm{Ryd})$ \\
\hline OF-AIMD & 943 & 500 & 0.05290 & 30.25 \\
OF-AIMD & 1323 & 500 & 0.05071 & 29.25 \\
LRT-CMD & 943 & 600 & 0.05290 & \\
LRT-CMD & 1323 & 500 & 0.05071 & \\
QHNC-CMD & 933 & 800 & 0.05331 & \\
\hline \hline
\end{tabular}

$$
E_{\mathrm{ext}}[\rho]=\int d \vec{r} \rho(\vec{r}) V_{\mathrm{ext}}(\vec{r}),
$$

the classical electrostatic energy (Hartree term)

$$
E_{H}[\rho]=\frac{1}{2} \iint d \vec{r} d \vec{s} \frac{\rho(\vec{r}) \rho(\vec{s})}{|\vec{r}-\vec{s}|}
$$

and the exchange-correlation energy $E_{\mathrm{xc}}[\rho]$, for which we will adopt the local density approximation.

\section{A. Technical details}

Given an explicit functional $T_{s}[\rho]$, we can proceed to minimize $E_{g}$ with respect to $\rho(\vec{r})$; however, in order to maintain $\rho(\vec{r}) \geqslant 0$ everywhere, as our system variable we have used an effective orbital $\psi(\vec{r})$ defined as $\rho(\vec{r})=\psi(\vec{r})^{2}$, with real $\psi(\vec{r})$. We expand $\psi(\vec{r})$ in plane waves compatible with the simple cubic periodic boundary conditions of the simulation:

$$
\begin{gathered}
\psi(\vec{r})=\sum_{\vec{G}} c_{\vec{G}} e^{-i \vec{G} \cdot \vec{r}}, \\
c_{\vec{G}}=\frac{1}{V} \int_{V} d \vec{r} \psi(\vec{r}) e^{i \vec{G} \cdot \vec{r}}, \\
\vec{G}=\frac{2 \pi}{L}\left(n_{1}, n_{2}, n_{3}\right),
\end{gathered}
$$

where $L$ stands for the side of the cube. This expansion is truncated at wave vectors corresponding to a given cutoff energy, $E_{\text {Cut }}$, whose value is given in Table I. A real $\psi$ implies that $c_{-\vec{G}}=c_{\vec{G}}^{*}$, with a real $c_{0}$; consequently only the half-set $\left\{c_{\vec{G}}\right\}^{\prime}$ s need be treated as variables.

The energy functional must be minimized with the normalization constraint $\mathcal{G}[\rho(\vec{r})]=\int_{V} d \vec{r} \rho(\vec{r})=N_{\mathrm{e}}$ which is imposed via the Lagrange multiplier $\mu$, leading to the EulerLagrange equation

$$
\frac{\delta \mathcal{F}}{\delta \rho(\vec{r})} \equiv \frac{\delta[E-\mu \mathcal{G}]}{\delta \rho(\vec{r})}=\frac{\delta E}{\delta \rho(\vec{r})}-\mu \equiv \mu(\vec{r})-\mu=0
$$

for the ground-state density. The minimization is performed with respect to the $\left\{c_{\vec{G}}\right\}^{\prime} \mathrm{s}$, instead of the electronic density, leading to the equations

$$
\begin{gathered}
\frac{\partial \mathcal{F}}{\partial c_{0}}=2 \int_{V} d \vec{r} \mu(\vec{r}) \psi(\vec{r})-2 \mu V c_{0}=0, \\
\frac{\partial \mathcal{F}}{\partial c_{\vec{G}}}=4 \int_{V} d \vec{r} \mu(\vec{r}) \psi(\vec{r}) e^{i \vec{G} \cdot \vec{r}}-4 \mu V c_{\vec{G}}=0
\end{gathered}
$$

for the ground-state density. The minimization of the functional is performed every time step of the simulation, using a simple quenching method: a fictitious "coefficients' kinetic energy" $\mathcal{T}=\frac{1}{2} M_{c} \Sigma_{\vec{G}}\left|\dot{c}_{\vec{G}}\right|^{2}$ is introduced, where $M_{c}$ is the "coefficients' mass," and the dot denotes the derivative with respect to the fictitious "coefficients' time" $t_{c}$. This kinetic energy, rewritten in terms of the set $\left\{c_{\vec{G}}\right\}$, together with the "potential energy" $\mathcal{F}$, leads to the following "equation of motion" $\left(\forall c_{\vec{G}} \in\left\{c_{\vec{G}}\right\}\right)$ :

$$
M_{c} \ddot{c}_{\vec{G}}=-2 \int_{V} d \vec{r} \mu(\vec{r}) \psi(\vec{r}) e^{i \vec{G} \cdot \vec{r}}+2 \mu V c_{\vec{G}} .
$$

These equations are solved numerically using the Verlet leapfrog algorithm ${ }^{34}$ with an electronic time step $\Delta t_{c}$. The velocities are quenched at every step until the minimum is reached within preset tolerances on $\mathcal{T}$ and the gradient of $\mathcal{F}$. The chemical potential $\mu$ is not known in advance of the minimization, but replacing $\mu$ in Eq. (15) by its stationary value $\int d \vec{r} \mu(\vec{r}) n(\vec{r}) / \int d \vec{r} n(\vec{r})$, at each time step, gives a good convergence to the ground state. For the present simulations, we have used $M_{c}=1.85 \times 10^{7}$ Hartree $\times(\text { a.u. })^{3}$ and a $\Delta t_{c}$ $=1 \times 10^{-4} \mathrm{ps}$.

The interatomic forces are obtained from the electronic ground state via the Hellman-Feynman theorem $\vec{F}_{i}$ $=-\vec{\nabla}_{\vec{R}_{i}} E_{g}\left[\rho(\vec{r}),\left\{\vec{R}_{l}\right\}\right],(i=1, \ldots, N)$, and Newton's equations $d^{2} \vec{R}_{i} / d t^{2}=\vec{F}_{i} / M_{i}$ are solved numerically for the motion of the ions using the Verlet leapfrog algorithm with a time step of $\Delta t=1.5 \times 10^{-3}$ ps.

\section{B. Kinetic-energy functional}

The kinetic-energy functional $T_{s}$ is a critical ingredient of the energy functional. It is generally considered ${ }^{35}$ that the von Weizsäcker term

$$
T_{W}[\rho(\vec{r})]=\frac{1}{8} \int d \vec{r}|\nabla \rho(\vec{r})|^{2} / \rho(\vec{r})
$$

is essential for a good description of the kinetic energy. It applies in the case of rapidly varying densities, and it is exact for one- or two-electron systems. Further terms are usually added to the functional in order to reproduce correctly some exactly known limits. In the uniform density limit, the exact kinetic energy is given by the Thomas-Fermi functional

$$
T_{\mathrm{TF}}[\rho(\vec{r})]=\frac{3}{10} \int d \vec{r} \rho(\vec{r}) k_{F}(\vec{r})^{2},
$$

where $k_{F}(\vec{r})=\left(3 \pi^{2}\right)^{1 / 3} \rho(\vec{r})^{1 / 3}$ is the local Fermi wave vector. In the limit of almost uniform density, the LRT is correct, 
with a response function corresponding to a noninteracting uniform electron gas, given by the Lindhard function $\chi_{L}\left(q, \rho_{0}\right)$.

Stimulated by the advantages of the orbital-free $a b$ initio simulations, there has been a renewed interest in the development of accurate kinetic-energy functionals. With Perrot's work as the basis, ${ }^{36}$ Madden and co-workers ${ }^{37,38}$ developed functionals which correctly recover the Thomas-Fermi and linear-response limits, ${ }^{37}$ and also included the quadratic response. ${ }^{38}$ Later, Wang et al. ${ }^{39}$ investigated these functionals and proposed a linear combination of them as a suitable form for $T_{s}$; more recently they also derived another expression which includes density-dependent kernels. Unfortunately, an undesirable feature of these functionals is that they are not positive definite, so that a minimization of the energy functional can lead to an unphysical negative kinetic energy.

Chacón, Alvarellos, and Tarazona ${ }^{40}$ developed a different type of kinetic-energy functional, which employs an "averaged density" and recovers the uniform and LRT limits. Their functional was investigated and generalized by García-González et al. ${ }^{41}$ These functionals have the merit of being positive definite, but they are somewhat complicated to apply and require order $N$ more fast Fourier transforms (FFT's) than simpler functionals, and this diminishes the advantage of the orbital-free approach over the full Kohn-Sham method.

In this paper we use a simplification of the averaged density approach, ${ }^{41}$ with the kinetic energy given by

$$
\begin{gathered}
T_{s}=T_{W}[\rho]+T_{\beta}[\rho], \\
T_{\beta}[\rho]=\frac{3}{10} \int d \vec{r} \rho(\vec{r})^{5 / 3-2 \beta} \widetilde{k}(\vec{r})^{2}, \\
\widetilde{k}(\vec{r})=\left(2 k_{F}^{0}\right)^{3} \int d \vec{s} k(\vec{s}) w_{\beta}\left(2 k_{F}^{0}|\vec{r}-\vec{s}|\right), \\
k(\vec{r})=\left(3 \pi^{2}\right)^{1 / 3} \rho(\vec{r})^{\beta},
\end{gathered}
$$

where $k_{F}^{0}$ is the Fermi wave vector corresponding to a mean electron density $\rho_{0}$, and $w_{\beta}(x)$ is a weighting function, determined by requiring the correct recovery of the LRT and uniform density limits. Note that $\widetilde{k}(\vec{r})$ appears as a convolution which can be performed rapidly by the usual FFT techniques. This functional is a generalization of one with $\beta$ $=1 / 3$, used earlier by us in a study of expanded liquid Cs. ${ }^{27}$

The details of the functional are given in Appendix A, and its main characteristics are as follows: (i) $\beta$ is a real positive number whose maximum value leading to a mathematically well behaved weight function is $\approx 0.6$. (ii) The functional recovers the uniform and LRT limits, and is positive definite. (iii) When $k_{F}^{0} \rightarrow 0$, because the mean electron density vanishes; e.g. for a finite system, the von Weizsäcker term is recovered if $\beta=4 / 9$, whereas for other values of $\beta$, the limit is $T_{W}+C T_{\mathrm{TF}}$. (iv) For values of $\beta>0.5$ it is expected that $\mu(\vec{r}) \psi(\vec{r})$, which is the driving force for the dynamic minimization of the energy [see Eq. (15)], remains finite even for very small electronic densities $\rho(\vec{r})$.
Those two last properties will be important in the case of expanded liquid metals because of the appearance of large inhomogeneities in the atomic distribution, and therefore in the electron density, with regions where it becomes very small. Indeed, this situation has already been observed in the $a b$ initio simulations of expanded liquid $\mathrm{Na}^{20}$ In systems for which the appearance of isolated atoms or clusters is likely, the von Weizsäcker term would be appropriate, and a functional with a value of $\beta$ as close as possible to $4 / 9$ would be recommended.

In the present simulations we have used $\beta=0.51$, which in the limit $\rho_{0} \rightarrow 0$ gives $C=0.046$ and guarantees, at least for the thermodynamic states considered, that $\mu(\vec{r}) \psi(\vec{r})$ remains finite and not too large everywhere so that the energy minimization can be achieved.

\section{Pseudopotentials}

$A b$ initio simulations using the full Kohn-Sham approach (KS-AIMD) usually employ nonlocal pseudopotentials obtained by fitting to some properties of the free atom. ${ }^{42}$ In an orbital-free approach where the electronic density is the variable, such nonlocal pseudopotentials, which act differently on different angular momentum components of the orbitals, cannot be used. Instead, local pseudopotentials must be developed which include an accurate description of the electronic structure in the physical circumstances of interest.

When constructing a pseudopotential to be used for a liquid metal, it seems more appropriate to use a reference state which closely resembles the environment of an atom in the metal, which is quite different from free space. The pseudopotential used in this simulation has been obtained using the neutral pseudoatom method ${ }^{43}$ in which the reference state is an atom at the center of a spherical cavity in the positive background of a uniform electron gas. The density of the gas is taken to be the mean valence electron density of the system of interest, in our case the liquid metal in a specific thermodynamic state. The radius of the cavity is such that the total positive charge removed from the hole is equal to the valence of the atom. First, a full Kohn-Sham density functional (KS-DFT) calculation is performed to obtain the displaced valence electron density $n_{\mathrm{ps}}(r)$, i.e., the change in the electron density induced by the atom and the cavity. After pseudizing $n_{\mathrm{ps}}(r)$ by eliminating the core-orthogonality oscillations, an effective local pseudopotential is constructed which, when inserted into the uniform electron gas along with the cavity, reproduces the displaced valence electron density previously obtained.

Two approaches will be followed in this paper. The first uses LRT to reproduce the displaced valence electron density obtained by the KS-DFT calculation, leading to a LRT-based local pseudopotential (LRT-PS) from which an effective interatomic pair potential is derived [see Eq. (6)] to be used in CMD simulations; for further details we refer to Ref. 43. The second approach uses the orbital-free density funtional theory (OF-DFT) to reproduce the displaced electron density obtained by the KS-DFT calculation, and it is suited for OFAIMD simulations. The development proceeds as follows (further details are given in Appendix C). When the func- 
tional derivatives of the energy functional are performed, the Euler equation [Eq. (13)], for our pseudopotential in the jellium-vacancy system becomes

$$
\mu_{s}(r)+V_{\text {ext }}(r)+V_{H}(r)+V_{\mathrm{xc}}(r)-\mu=0,
$$

where each of the terms is the derivative of the corresponding term in Eq. (7), namely,

$$
\mu_{s}(r)=\mu_{W}(r)+\mu_{\beta}(r),
$$

with the expressions for the von Weizsäcker term and the $\beta$ term given in Appendix B,

$$
\begin{gathered}
V_{\text {ext }}(r)=v_{\mathrm{ps}}(r)+v_{\mathrm{cav}}(r)+v_{\text {jell }}(r), \\
V_{H}(r)=\int d \vec{s} \rho(s) /|\vec{r}-\vec{s}|,
\end{gathered}
$$

with $\rho(r)=\rho_{0}+n_{p s}(r)$, and $V_{\mathrm{xc}}(r)$ is the exchangecorrelation potential, obtained from the functional derivative of $E_{\mathrm{xc}}[\rho]$ evaluated at $\rho(r)$.

Due to the spherical symmetry of the system all the magnitudes depend just on $r$. Given $\rho(r), v_{\mathrm{ps}}(r)$ can be obtained from Eq. (22), and the constant $\mu$ is just an energy origin which is set so as to obtain a pseudopotential that decays to zero for large distances. The pseudopotential constructed in this way, will be referred to as the OF-DFT-based pseudopotential (OFDFT-PS). Note that the pseudopotential is calculated for arbitrary $r$, and therefore, upon Fourier transformation, for arbitrary $q$. A similar procedure was already suggested, ${ }^{45}$ although using a crystal as the reference state. This choice has the disadvantage that the pseudopotential is obtained only at the Bragg vectors of the reciprocal lattice, and not for arbitrary $q$ values.

\section{RESULTS AND DISCUSSION}

We have performed OF-AIMD simulations for liquid Al at two different thermodynamic states along the liquid-vapor coexistence line (943 and $1323 \mathrm{~K}$ ), for which X-ray- and neutron-diffraction data are available. ${ }^{29-32}$ Table I gives further details on the thermodynamic states and several simulation parameters. In addition, we have also carried out classical MD simulations, using effective interionic pair potentials derived from standard second order pseudopotential perturbation theory, with the LRT-PS's constructed as previously described (also see Ref. 43) and with pair potentials derived from the QHNC method.

In the OF-AIMD simulations 500 particles were treated in a cubic cell of the size appropriate to the density, whereas more particles were used for the CMD simulations (see Table I). In both sets of simulations, liquid static properties were evaluated (pair distribution functions and static structure factors) as well as several dynamic properties, both singleparticle ones (velocity autocorrelation function, mean square displacement) and collective ones (intermediate scattering functions, dynamic structure factors, longitudinal and transverse currents). The calculation of the collective dynamic properties required long simulation runs in order to accumulate reasonable statistics; for example the OF-AIMD simula-

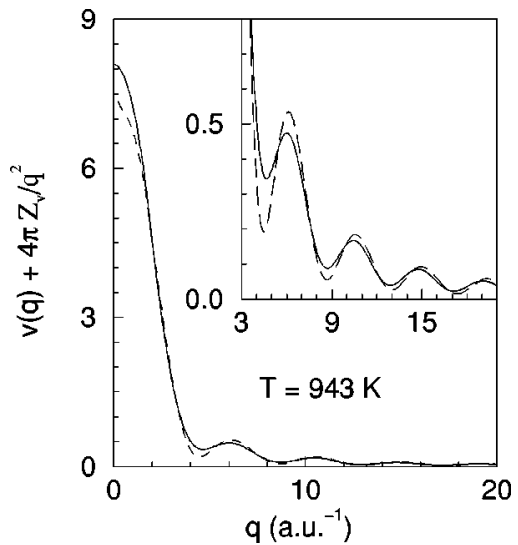

FIG. 1. Non-Coulombic part of the pseudopotential for Al at $T$ $=943 \mathrm{~K}$. The continuous line is the OFDFT-PS used in the OFAIMD simulations, while the dashed line stands for the LRT-PS used for the CMD simulations.

tions lasted for $2 \times 10^{4}$ steps, which correspond to $30 \mathrm{ps}$ of simulation time. On the other hand, the CMD simulations run for $10^{5}$ steps, amounting to 200 ps.

\section{A. Pseudopotentials}

The local pseudopotentials described in Sec. II C were constructed using a reference system mimicking the complex system to be studied. The pseudopotentials change with the thermodynamic state considered and therefore are not transferable to other states. Figure 1 shows the Fourier transforms of the non-Coulombic part of the pseudopotentials obtained from the LRT and OF-DFT approaches outlined above. The two schemes lead to similar pseudopotentials, with the main differences being at low- $q$ values and in the amplitude of the oscillations at large $q$. Note that in both approaches the same pseudized displaced valence electronic density of an atom in a jellium-vacancy model is reproduced, although OF-DFT was used in one case and LRT in the other. Consequently the differences in the two pseudopotentials should reflect the importance of nonlinear effects which, according to the present results, seem to be more important at small $q$. The appearance of the oscillations can be traced back to the calculation of the pseudized displaced valence electronic density which has a discontinuous second derivative at a matching radius. However, these oscillations do not influence the final OFAIMD results because they appear for $q$ values larger than those corresponding to $E_{\mathrm{Cut}}$.

\section{B. Static properties}

The static structure factors $S(q)$ obtained from the simulations are shown in Fig. 2, which also shows the corresponding experimental data measured by neutron (Ref. 30) and $\mathrm{x}$-ray-diffraction ${ }^{31,32}$ experiments. The experimental data show small differences in the region $2 \AA^{-1} \leqslant q \leqslant 5 \AA^{-1}$, with the neutron values being slightly bigger that the $\mathrm{X}$-ray ones, whereas the OF-AIMD results stand remarkably well between both sets, although somewhat closer to the x-ray data. The insets of the figures show that the OF-AIMD results in the small- $q$ region are also in good agreement with 


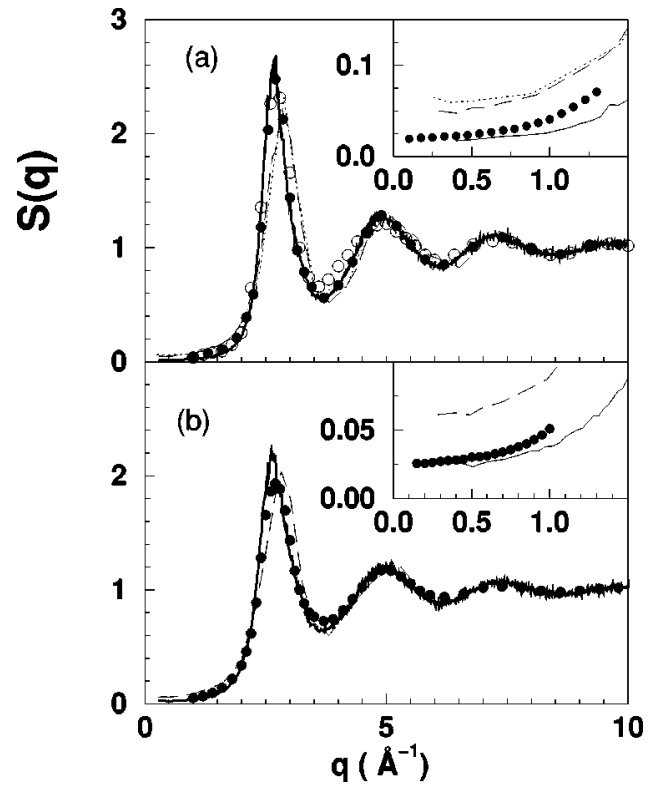

FIG. 2. Static structure factors of liquid $\mathrm{Al}$ at (a) $943 \mathrm{~K}$ and (b) 1323 K. Full circles: experimental x-ray-diffraction data (Ref. 31 and 32). Open circles: experimental neutron-diffraction data (Ref. 30). Continuous line: OF-AIMD simulations. Dashed lines: LRTCMD simulations. Dotted lines: CMD simulations with the QHNC potential. The insets show the low- $q$ behavior.

the experimental x-ray results. The figures also include $S(q)$, obtained from the CMD simulations performed with the interatomic pair potential derived from the LRT-PS and QHNC methods. ${ }^{14,15}$ Although these calculated $S(q)$ 's reasonably reproduce the experimental data, the agreement with experiment is much better for OF-AIMD results.

Extrapolation of $S(q)$ to $q \rightarrow 0$ allows the isothermal compressibility $\kappa_{T}$ to be estimated from the relation $S(0)$ $=\rho k_{B} T \kappa_{T}$. A least-squares fit of $S(q)=s_{0}+s_{2} q^{2}$ to the calculated $S(q)$ for $q$ values up to $1 \AA^{-1}$ yields the result $\kappa_{T, \mathrm{OF}-\mathrm{AIMD}}=2.37$ (in $10^{-11} \mathrm{~m}^{2} \mathrm{Nw}^{-1}$ units) for $T$ $=943 \mathrm{~K}$, which is close to the experimental value ${ }^{44} \kappa_{T}$ $=2.43$. In contrast, both the LRT-PS and QHNC interionic pair potentials lead to much higher values, namely, $\kappa_{T, \mathrm{LRT}-\mathrm{CMD}}=6.5$ and $\kappa_{T, \mathrm{QHNC}-\mathrm{CMD}}=7.4$, respectively.

The ionic and electronic static structure of liquid Al near melting was also calculated by Anta et $a .^{26}$ using the OFAIMD method with a kinetic-energy functional which describes the correct linear and quadratic response of the electron gas $^{38}$ and a local ionic pseudopotential constructed from a nonlocal ionic pseudopotential. ${ }^{26,45}$ Their results for the static structure factor closely followed the experimental one.

\section{Dynamic properties}

\section{Single-particle dynamics}

The most complete information about the single-particle properties is provided by the self-intermediate scattering function $F_{s}(q, t)$, which probes the single-particle dynamics over different length scales, ranging from the hydrodynamic limit $(q \rightarrow 0)$ to the free-particle limit $(q \rightarrow \infty)$. In the present simulations, this magnitude has been obtained by
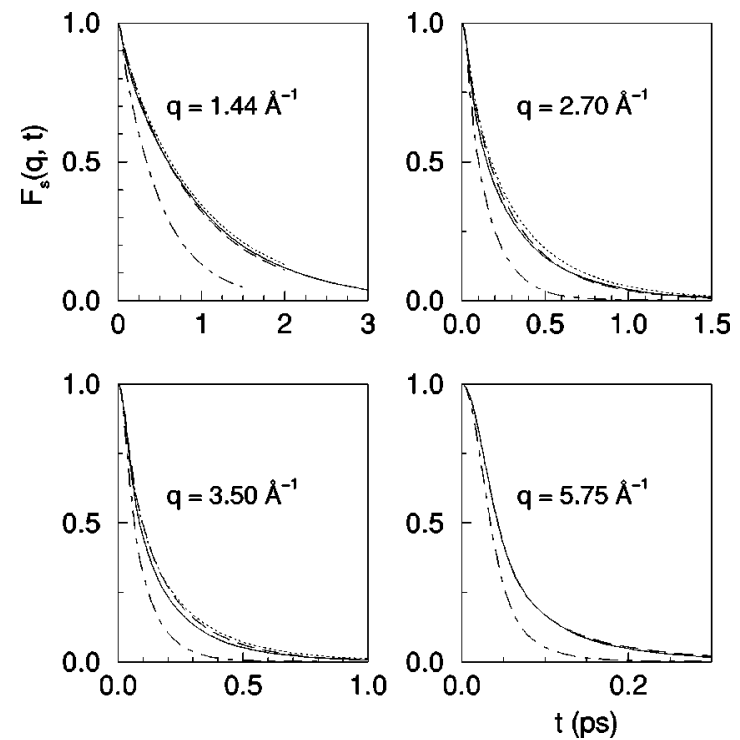

FIG. 3. Self-intermediate scattering functions $F_{s}(q, t)$ at several $q$ values, for liquid aluminum. Continuous, dashed, and dotted lines: OF-AIMD, LRT-CMD, and QHNC-CMD simulations, respectively, at $T=943 \mathrm{~K}$. Dash-dotted line: OF-AIMD simulations at $T=1323 \mathrm{~K}$.

$$
F_{s}(q, t)=\frac{1}{N}\left\langle\sum_{j=1}^{N} e^{-i \vec{q} \vec{R}_{j}\left(t+t_{0}\right)} e^{i \vec{q} \vec{R}_{j}\left(t_{0}\right)}\right\rangle
$$

and in Fig. 3 we show the results obtained for several $q$ values at $T=943$ and $1343 \mathrm{~K}$. This shows the typical monotonic decrease with time; moreover, the results are very similar to those of the LRT-CMD and QHNC-CMD simulations, although the latter show a slightly slower decay with time. An increase in temperature leads to increased rate of decay.

Closely related to the $F_{s}(q, t)$ is the velocity autocorrelation function (VACF) of a tagged ion in the fluid, $Z(t)$, which can be obtained as the $q \rightarrow 0$ limit of the first-order memory function of the $F_{s}(q, t)$. However, in the present simulations it is more easily obtained from its definition

$$
Z(t)=\left\langle\vec{v}_{1}(t) \vec{v}_{1}(0)\right\rangle /\left\langle v_{1}^{2}\right\rangle
$$

which stands for the normalized VACF. The results are shown in Fig. 4 along with those derived from the LRT-CMD and QHNC-CMD simulations. The results display the typical

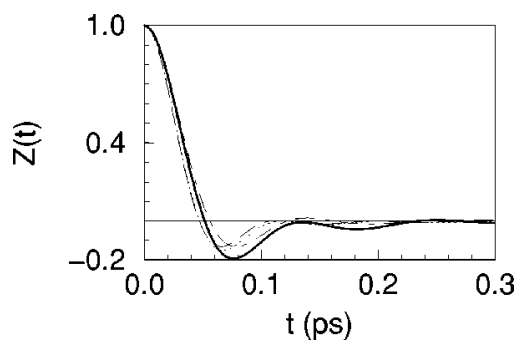

FIG. 4. Normalized velocity autocorrelation functions. Continuous, dashed, and dotted lines: OF-AIMD, LRT-CMD, and QHNCCMD simulations, respectively, at $T=943 \mathrm{~K}$. Dash-dotted line: OF-AIMD results for $T=1323 \mathrm{~K}$. 
TABLE II. Isothermal compressibility $\kappa_{T}$ (in $10^{-11} \mathrm{~m}^{2} \mathrm{~N}^{-1}$ ), self-diffusion coefficient $D$ (in $\AA^{2} / \mathrm{ps}$ ), and shear viscosity coefficient $\eta$ (in GPa ps) of liquid $\mathrm{Al}$ at the thermodynamic states studied in this work.

\begin{tabular}{lcccc}
\hline \hline & $T(\mathrm{~K})$ & $\kappa_{T}$ & $D$ & $\eta$ \\
\hline OF-AIMD & 943 & 2.37 & 0.49 & 1.38 \\
OF-AIMD & 1323 & 2.38 & 1.05 & 0.85 \\
LRT-CMD & 943 & 6.57 & 0.58 & 1.24 \\
LRT-CMD & 1323 & 6.32 & 1.14 & - \\
QHNC-CMD & 933 & 7.45 & 0.55 & 1.36 \\
Experiment & 933 & $2.43^{\mathrm{a}}$ & & $1.26^{\mathrm{b}}$ \\
\hline \hline
\end{tabular}

${ }^{\mathrm{a}}$ Reference 44.

${ }^{\mathrm{b}}$ Reference 70 .

backscattering behavior, which is more marked for the OFAIMD simulations, but the results of the three simulations are rather similar. The main features of the obtained $Z(t)$ are comparable to those obtained for other simple metals near melting, ${ }^{46-49}$ namely (i) a first minimum about 0.20 deep, and (ii) a rather weak following maximum peaking close to zero. The self-diffusion coefficient $D$ is readily obtained from either the time integral of $Z(t)$ or from the slope of the mean-square displacement $\delta R^{2}(t) \equiv\left\langle\left|\vec{R}_{1}(t)-\vec{R}_{1}(0)\right|^{2}\right\rangle$ of a tagged ion in the fluid, as

$$
D=\frac{1}{\beta m} \int_{0}^{\infty} Z(t) d t, \quad D=\lim _{t \rightarrow \infty} \delta R^{2}(t) / 6 t
$$

and the results for $D$ are given in Table II. The two routes for $D$ lead to practically the same value, namely, $D_{\text {OF-AIMD }}$ $=0.49 \AA^{2} / \mathrm{ps}$; which is somewhat smaller than the mean value of $0.55 \AA^{2} /$ ps obtained in a previous OF-AIMD calculation with 205 particles. $^{25}$ Unfortunately, to our knowledge, no experimental results are yet available for the diffusion coefficients of liquid $\mathrm{Al}$ at any thermodynamic state. However, we can compare with the results of a KS-DFT calculation $^{50}$ performed for liquid $\mathrm{Al}$ near the tripe point, using 64 particles and a nonlocal Bachelet-Hamann-Schluter type pseudopotential; ${ }^{51-53}$ this calculation lead to a value $D_{\mathrm{KS}-\mathrm{DFT}}=0.60 \AA^{2} / \mathrm{ps}$ derived from the slope of the corresponding mean square displacement. Recently, another KSDFT calculation $^{54}$ for liquid $\mathrm{Al}$ at $1000 \mathrm{~K}$, using 64 particles and ultrasoft Vanderbilt pseudopotentials gave $D$ values within the range $0.52-0.68 \AA^{2} / \mathrm{ps}$, derived from the slope of the mean-square displacement. Our OF-AIMD simulations, with a small number of particles and/or a small number of configurations, suggest that the self-diffusion coefficients obtained from the $\delta R^{2}(t)$ tend to be greater than those obtained by integration of the $Z(t)$, and, as the number of particles and/or configurations is increased, the value for the selfdiffusion coefficient is decreased. More extensive KS-DFT simulations would probably lead to a smaller value of $D$ closer to that obtained in the present OF-AIMD simulations. The values obtained from LRT-CMD $\left(0.58 \AA^{2} / \mathrm{ps}\right)$ and QHNC-CMD (0.55 $\left.\AA^{2} / \mathrm{ps}\right)$ simulations are also rather similar, and slightly larger than the OF-AIMD result. The CMD

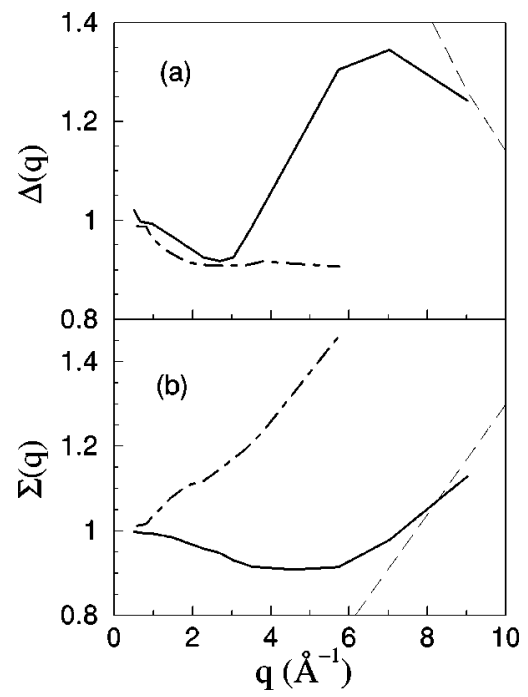

FIG. 5. (a) Normalized half-width of $S_{s}(q, \omega)$, relative to its value at the hydrodynamic limit, for liquid aluminum at $T$ $=943 \mathrm{~K}$ (continuous line) and $1323 \mathrm{~K}$ (dash-dotted line). The dashed line stands for the free-particle limit. (b) Same as before, but for the normalized peak value $S_{s}(q, \omega=0)$, relative to its value at the hydrodynamic limit.

simulations of Ebbsjo et al., ${ }^{7}$ using several pair potentials, gave values within the range $0.41-0.45 \AA^{2} / \mathrm{ps}$.

By Fourier transforming $F_{s}(q, t)$ we obtain the selfdynamic structure factor $S_{s}(q, \omega)$, which, for all $q$ values, exhibits a monotonic decay with frequency, from a peak value at $\omega=0 . S_{s}(q, \omega)$ can be characterized by the peak value $S_{s}(q, \omega=0)$, and the half-width at half-maximum $\omega_{1 / 2}(q)$. These parameters are usually reported normalized with respect to the values of the hydrodynamic $(q \rightarrow 0)$ limit, by introducing the dimensionless quantities $\Sigma(q)$ $=\pi q^{2} D S_{s}(q, \omega=0) \quad$ and $\quad \Delta(q)=\omega_{1 / 2}(q) / q^{2} D, \quad$ where $\omega_{1 / 2}(q) / q^{2}$ can be interpreted as an effective $q$-dependent diffusion coefficient $D(q)$. For a liquid near the triple point, $\Delta(q)$ usually exhibits an oscillatory behavior whereas in a dense gas it decreases monotonically from unity at $q=0$ to the $1 / q$ behavior at large $q$. Figure 5 shows the OF-AIMD results for $\Delta(q)$ and $\Sigma(q)$; the corresponding results from the LRT-CMD and QHNC-CMD simulations are rather similar and are not shown. The results for $\Delta_{\text {OF-AIMD }}(q)$, show that, for both temperatures, the hydrodynamic limit is reached from below, with a minimum at around $q \approx q_{p}$, followed by a maximum and by a gradual transition, for greater $q$ values, to the free-particle limit. Note that, for the higher temperature, the oscillations are heavily damped and the free particle limit is approached quickly. This oscillating behavior of $\Delta_{\text {OF-AIMD }}(q)$ for small and intermediate $q$ values was reported by several authors, and attributed to the coupling of the single-particle motion to other modes in the system. ${ }^{4,55-59}$ On the other hand, the results for $\Sigma(k)$ reflect greater sensitivity to changes in temperature, with the diffusive limit reached from below for $T=943 \mathrm{~K}$ and from above for $T=1323 \mathrm{~K}$. We note that similar features to those obtained in this paper were obtained earlier by Torcini et al. ${ }^{60}$ 

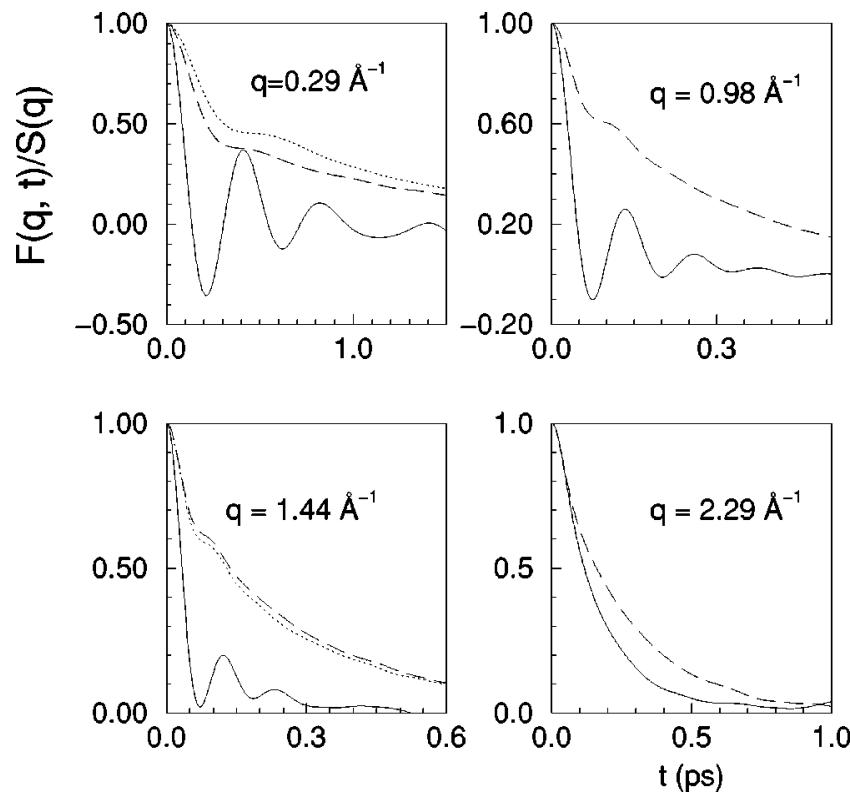

FIG. 6. Normalized intermediate scattering functions $F(q, t)$ at several $q$ values, for liquid aluminum at $T=943 \mathrm{~K}$. Continuous line: OF-AIMD simulations. Dashed line: LRT-CMD results. Dotted line: QHNC-CMD results.

in their CMD study of liquid lithium near melting using the interatomic pair potential proposed by Price et $a l^{61}$

\section{Collective dynamics}

The intermediate scattering function $F(q, t)$ embodies the information concerning the collective dynamics of density fluctuations over both the length and time scales. It is defined as

$$
F(q, t)=\frac{1}{N}\left\langle\left(\sum_{j=1}^{N} e^{-i \vec{q} \vec{R}_{j}\left(t+t_{0}\right)}\right)\left(\sum_{l=1}^{N} e^{i \vec{q} \vec{R}_{l}\left(t_{0}\right)}\right)\right\rangle .
$$

In Figs. 6 and 7 we show the results from the present OFAIMD simulations for several $q$ values. $F(q, t)$ exhibits an oscillatory behavior which persists up to $q \approx 3 q_{p} / 5$, with the amplitude of the oscillations being stronger for the smaller $q$ values. This is typical behavior found for other simple liquid metals near melting, by either computer simulations ${ }^{60,62,63}$ or theory. ${ }^{64}$ A different behavior is seen for the results in the same $q$ range obtained from the LRT-CMD and QHNCCMD simulations, with $F(q, t)$ 's whose contact values, given by $F(q, t=0)=S(q)$, are more than double and, more important, with a diffusive component playing a dominant role. The corresponding MD results of Ebbjso et al. ${ }^{7}$ for the $F(q, t)$ 's have better contact values but also display an important diffusive component.

Closely connected to the $F(q, t)$ is the dynamic structure factor $S(q, \omega)$, which is obtained by a time Fourier transform of the $F(q, t)$ (with an appropriate window to smooth out truncation effects). Its importance lies in its direct connection to the inelastic neutron scattering or the IXS data. The results obtained for the $S(q, \omega)$ are shown in Figs. 8 and 9 for a range of wave vectors up to $\approx 2.5 q_{p}$. The dynamic structure
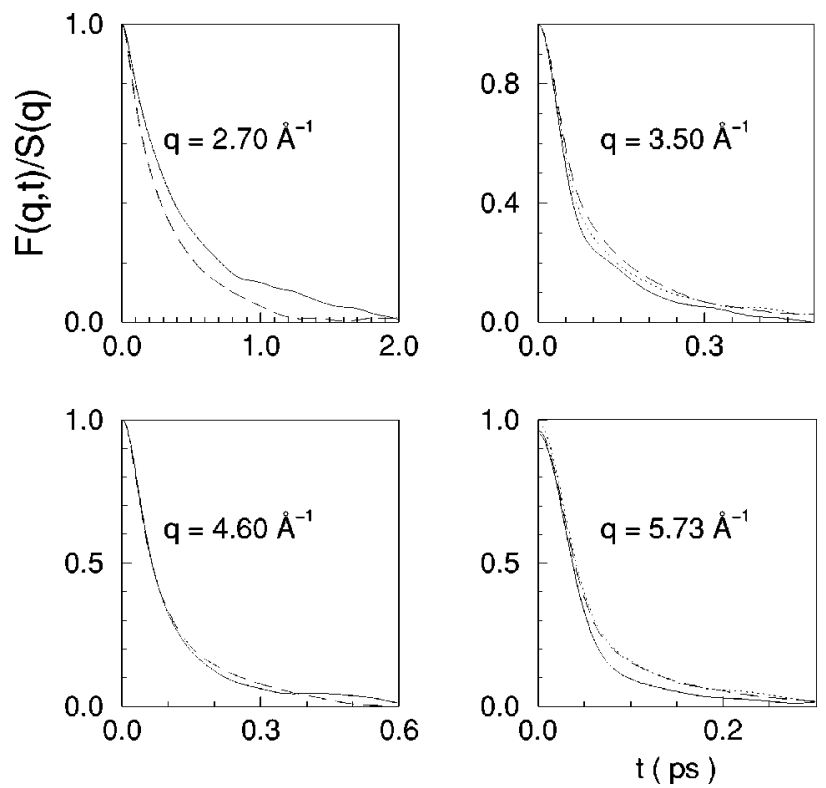

FIG. 7. Same as the previous figure.

factor shows well-defined side peaks, indicative of collective density excitations, up to $q \approx 1.6 \AA^{-1}$ which amounts to $\approx 3 q_{p} / 5$. The results qualitatively reproduce the shape of the experimental IXS data, ${ }^{33}$ although with some discrepancies in the heights and positions of the peaks. Similar results, but with a better description of the central peak at the lowest $q$ values, were also obtained in the CMD simulations of Ebbsjo et $a l{ }^{7}$ However, it must be stressed that their $F(q, t)$ 's were previously fitted to an analytical expression interpolating among the ideal gas, viscoelastic and hydrodynamic models,
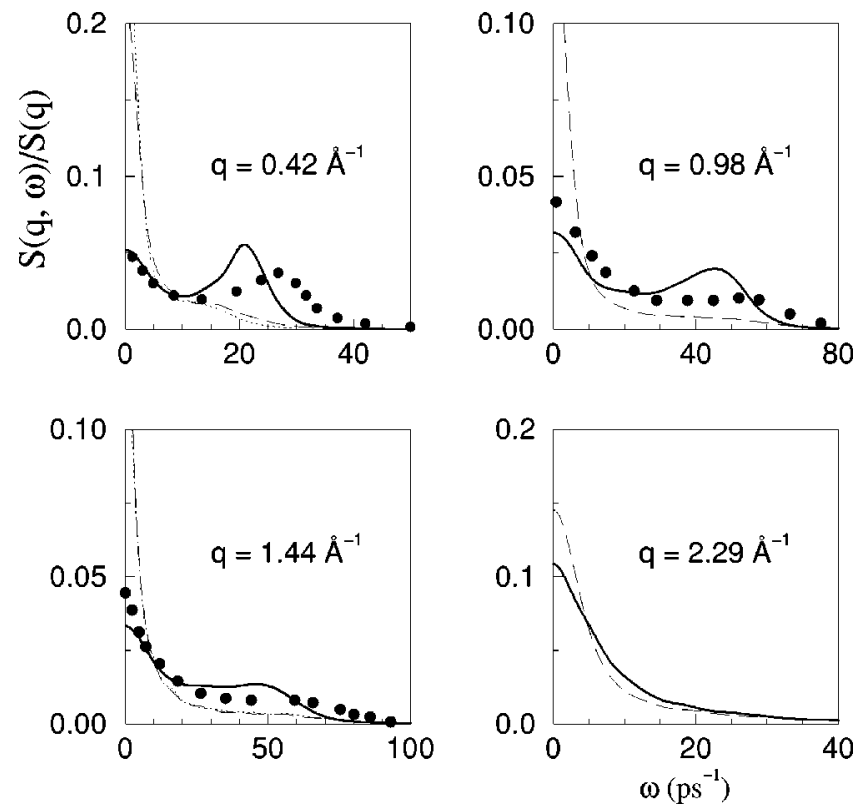

FIG. 8. Dynamic structure factor $S(q, \omega)$ for several $q$ values, for liquid aluminum at $T=943 \mathrm{~K}$. Continuous line: OF-AIMD simulations. Dashed line: LRT-CMD results. Dotted line: QHNCCMD results. Full circles: experimental data (Ref. 33) for $q=0.42$, 1.02 , and $1.38 \AA^{-1}$. 

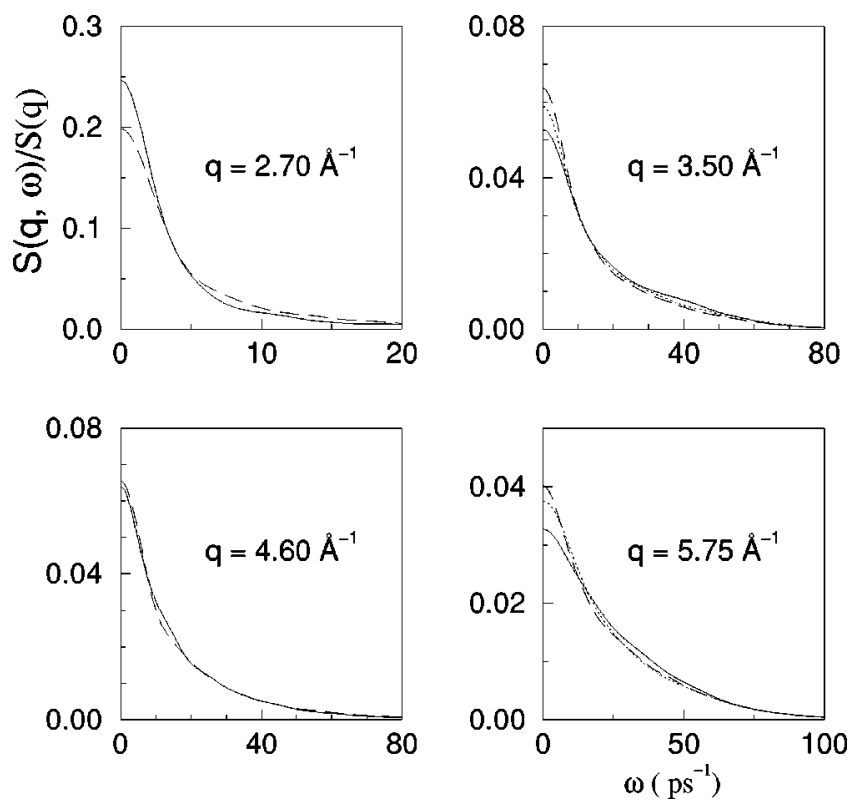

FIG. 9. Same as the previous figure.

and thus the corresponding $S(q, \omega)$ 's were derived. On the other hand, the strongly diffusive character of the $F(q, t)$ 's obtained from both the LRT-CMD and QHNC-CMD simulations, give rise to $S(q, \omega)$ which decay rather quickly, with hardly discernable side peaks. This is because the side peaks are located at smaller positions, given by $q c_{s}(q)$, where $c_{s}(q)$ is the generalized adiabatic sound velocity (see below), which is too small because of the large values of $S(q)$ at those $q$-values.

From the positions of the sidepeaks, $\omega_{m}(q)$, the dispersion relation of the density fluctuations has been obtained, and this is shown in Fig. 10 for the state at $T=943 \mathrm{~K}$, along with $\omega_{l}(q)$, which is the dispersion relation obtained from the maxima of the longitudinal current correlation function, $J_{l}(q, \omega)=\omega^{2} S(q, \omega)$. Note that in the hydrodynamic region (small $q$ ), the slope of the dispersion relation curve is the

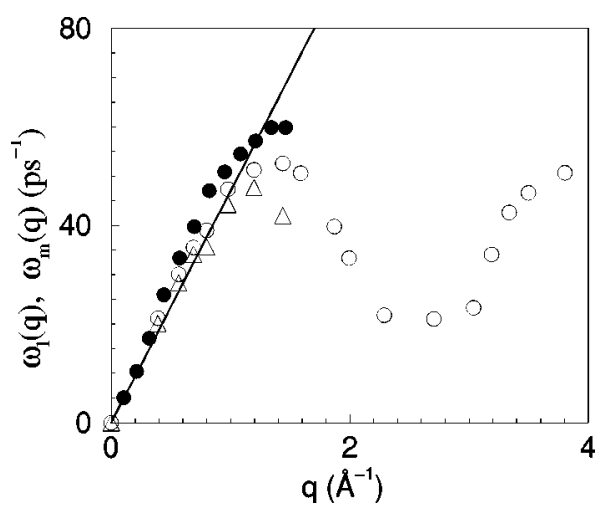

FIG. 10. Dispersion relation for liquid Al at $T=943 \mathrm{~K}$. Open triangles: peak positions $\omega_{m}(q)$ from the OF-AIMD $S(q, \omega)$. Open circles: peak positions $\omega_{l}(q)$ from the maxima of the OF-AIMD longitudinal current $J_{l}(q, \omega)$. Full circles: experimental $\omega_{l}(q)$ data from Scopigno et al. (Ref. 33). Full line: linear dispersion with the hydrodynamic sound velocity, $v=4700 \mathrm{~m} / \mathrm{s}$.

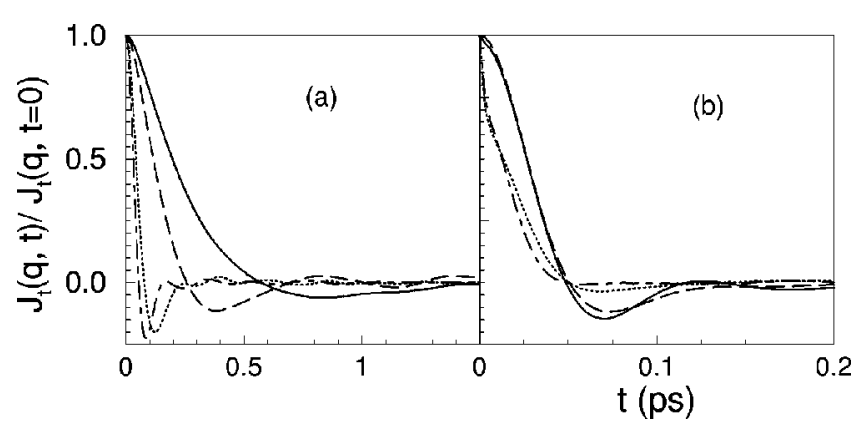

FIG. 11. OF-AIMF transverse current correlation function $J_{t}(q, t)$ at several $q$ values (in $\AA^{-1}$ ) for liquid $\mathrm{Al}$ at $T=943 \mathrm{~K}$. (a) $q=0.29$ (full curve), $q=0.42$ (dashed curve), $q=0.98$ (dotted curve), and $q=1.45$ (dash-dotted curve). (b) $q=2.70$ (full curve), $q=3.80$ (dashed curve), $q=5.54$ (dotted curve), and $q=9.02$ (dotdashed curve).

adiabatic sound velocity $c_{s}(q)=v_{t h} \sqrt{\gamma / S(q)}$, with $v_{t h}$ $=(\beta m)^{-1 / 2}$ being the thermal velocity and $\gamma$ the ratio of the specific heats. In the limit $q \rightarrow 0, c_{s}(q)$ reduces to the bulk adiabatic sound velocity and determines the slope of the dispersion at $q \rightarrow 0$. By extrapolating the OF-AIMD results for $S(q)$ and using the experimental value ${ }^{65}$ of $\gamma \approx 1.25$, we obtain a value of $\approx 4850 \mathrm{~m} / \mathrm{s}$ for the bulk adiabatic sound velocity which compares reasonably well with the experimental value 44 of $\approx 4700 \mathrm{~m} / \mathrm{s}$, near the triple point. Figure 10 shows a positive dispersion, i.e., an increase of $\omega_{l}(q)$ with respect to the values predicted by the hydrodynamic adiabatic speed of sound, with a maximum located around $0.4 \AA^{-1}$. A similar behavior was also obtained by Scopigno et $a l .{ }^{33}$ from their experimental IXS results for liquid $\mathrm{Al}$ at $T=1000 \mathrm{~K}$, and observed in other liquid metals: $\mathrm{Rb}, \mathrm{Cs}, \mathrm{Li}$ and $\mathrm{Na}$. ${ }^{33,66,67}$

Another interesting dynamical magnitude is the transverse current time correlation function $J_{t}(q, t)$, which is not associated with any measurable quantity and can only be determined by means of MD simulations. It provides information on the shear modes, and is defined as

$$
J_{t}(q, t)=\frac{1}{N}\left\langle j_{x}^{*}(q, 0) j_{x}(q, t)\right\rangle,
$$

where $j_{x}(q, t)=\sum_{j=1}^{N} v_{j}^{x}(t) e^{-i \vec{q} \vec{R}_{j}(t)}$ is the transverse current. The shape of $J_{t}(q, t)$ evolves from a Gaussian, in both $q$ and $t$, for the free-particle $q \rightarrow \infty$ limit, toward a Gaussian in $q$ and exponential in $t$ for the hydrodynamic limit $(q \rightarrow 0)$, i.e.,

$$
J_{t}(q \rightarrow 0, t)=\frac{1}{\beta m} e^{-q^{2} \eta|t| / m \rho},
$$

where $\eta$ is the shear viscosity. For intermediate $q$-values, $J_{t}(q, t)$ exhibits a more complicated behavior, as shown in Fig. 11 where OF-AIMD results for liquid Al near melting are shown. Note that for the smallest $q$ value reached by the simulation, $q=0.29 \AA^{-1}$, the corresponding $J_{t}(q, t)$ takes on negative values, which by Eq. (31) means that it is already beyond the hydrodynamic regime. The associated spectrum $J_{t}(q, \omega)$, plotted in Fig. 12, shows an inelastic peak which already exists at $q=0.29 \AA^{-1} \approx 0.11 q_{p}$; as $q$ in- 

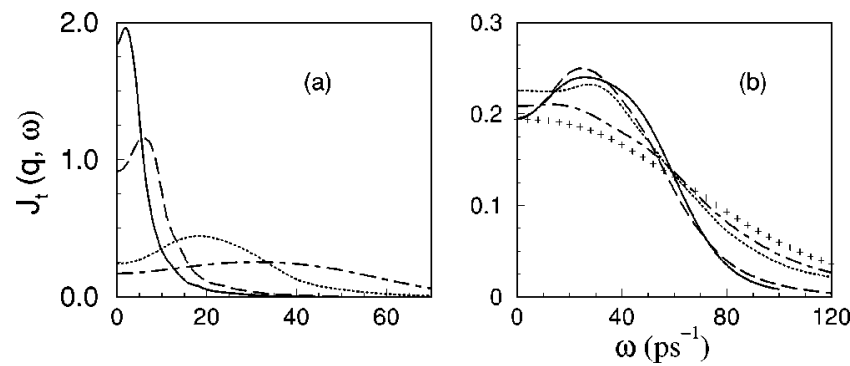

FIG. 12. OF-AIMF transverse current correlation spectrum $J_{t}(q, \omega)$ at several $q$ values (in $\AA^{-1}$ ) for liquid $\mathrm{Al}$ at $T=943 \mathrm{~K}$. (a) $q=0.29$ (full curve), $q=0.42$ (dashed curve), $q=0.98$ (dotted curve), and $q=1.99$ (dash-dotted curve). (b) $q=2.70$ (full curve), $q=3.80$ (dashed curve), $q=5.54$ (dotted curve), and $q=7.03$ (dotdashed curve), and $q=9.02$ (pulses).

creases the peak becomes better defined and it persists to $q$ values around $3 q_{p}$, although it has already disappeared for the largest $q$ value considered. Note that the associated peak frequency increases with $q$ up a maximum value at $q \approx q_{p}$, and then flattens at larger $q$ as $J_{t}(q, \omega)$ evolves toward a Gaussian shape. This behavior closely parallels that observed for the alkali metals, where the inelastic peak appears for $q$ $\geqslant 0.07 q_{p} \cdot{ }^{46}$

Similar results are also obtained by the LRT-CMD approach, but $J_{t}(q, t)$ decays more slowly and the minima are less marked. This leads to a spectrum $J_{t}(q, \omega)$ where the peaks are less marked and, in fact, there is no peak at $q$ $=0.29 \AA^{-1}$.

From the results for $J_{t}(q, t)$ we can readily obtain the shear viscosity coefficient $\eta$ as follows. ${ }^{46,68,69}$ The memory function representation of $J_{t}(q, t)$,

$$
\widetilde{J_{t}}(q, z)=\frac{1}{\beta m}\left[z+\frac{q^{2}}{\rho m} \tilde{\eta}(q, z)\right]^{-1}
$$

where the tilde denotes the Laplace transform, introduces a generalized shear viscosity coefficient $\tilde{\eta}(q, z)$. The area under the normalized $J_{t}(q, t)$, gives $\beta m \widetilde{J_{t}}(q, z=0)$, from which values for $\tilde{\eta}(q, z=0)$ can be obtained which, when extrapolated to $q=0$, give the usual shear viscosity coefficient $\eta$. Results for $\eta$ presented in Table II compare favorably with the available experimental data. ${ }^{70}$ For comparison, we note that the KS-DFT simulations of Alfe and Gillan ${ }^{54}$ gave values in the range 1.4-2.2 GPaps.

\section{CONCLUSIONS}

Several dynamic properties of liquid aluminum have been calculated at two thermodynamic states close to the triple point. Simulations have been performed using the orbital free $a b$ initio molecular-dynamics method, showing the feasibility of this technique to calculate several time correlation functions, allowing a comprehensive study of the dynamical properties. Furthermore, agreement with the available experimental data is reasonable.

We have also presented a method for producing, from first principles, local pseudopotentials for use with the orbital free-density functionals. While the ultimate goal of the method would be to use the atomic number of the atoms as the only input data, this has not yet been achieved, as the present calculations also require the experimental number density of the system for calculating the local pseudopotential and for performing the simulations. However, we stress that starting from very basic information, the present scheme allows the determination of the static and dynamic properties of the system.

Finally, we emphasize that in the present scheme, the calculation of the pseudopotential is coupled to the particular functional adopted for the total potential energy of the system. This means that different kinetic-energy functionals would lead to different pseudopotentials. Consequently, this field is open to further improvements in the description of the kinetic energy and, therefore, also in the corresponding local pseudopotential.

\section{ACKNOWLEDGMENTS}

This work was supported by the Junta de Castilla y León (Project No. VA70/99), NATO (CRG971173) and the DGES (PB98-0641-C02-01). D.J.G. acknowledges the UVA for the provision of financial support to visit the the Physics Dept. of Queen's University where part of this work was carried out. M.J.S. acknowledges the support of the NSERC of Canada.

\section{APPENDIX A: KINETIC-ENERGY FUNCTIONAL}

We consider the kinetic-energy functional

$$
T_{s}[\rho]=T_{W}[\rho]+T_{\beta}[\rho],
$$

where

$$
\begin{gathered}
T_{\beta}[\rho]=\frac{3}{10} \int d \vec{r} \rho(\vec{r})^{5 / 3-2 \beta} \widetilde{k}(\vec{r})^{2}, \\
\widetilde{k}(\vec{r})=\left(2 k_{F}^{0}\right)^{3} \int d \vec{s} k(\vec{s}) \omega_{\beta}\left(2 k_{F}^{0}|\vec{r}-\vec{s}|\right) \equiv k(\vec{r})^{*} \omega_{\beta}\left(2 k_{F}^{0} r\right), \\
k(\vec{r})=\left(3 \pi^{2}\right)^{1 / 3} \rho(\vec{r})^{\beta} .
\end{gathered}
$$

In the limit of small deviations from a uniform system, we wish to recover the LRT result. Equating the Fourier transform (FT) of the second functional derivative of $T_{s}[\rho]$ with respect to $\rho(\vec{r})$ for $\rho(\vec{r})=\rho_{0}$, to the inverse of the Lindhard response function, gives, for the weight function,

$$
\begin{gathered}
\left(6 \beta^{2}-\frac{20}{3} \beta+\frac{10}{9}\right)+4 \beta\left(\frac{5}{3}-2 \beta\right) \bar{\omega}_{\beta}(\eta)+2 \beta^{2} \bar{\omega}_{\beta}(\eta)^{2} \\
=\frac{10}{9}\left[1 / \pi_{L}(\eta)-3 \eta^{2}\right],
\end{gathered}
$$

where $\eta=q / 2 k_{F}^{0}, \bar{\omega}_{\beta}$ is the FT of $\omega_{\beta}$, and

$$
\pi_{L}(\eta)=\frac{1}{2}\left(1+\frac{1-\eta^{2}}{2 \eta} \ln \left|\frac{1+\eta}{1-\eta}\right|\right)
$$


is the noninteracting homogeneous electron-gas response function. Taking in Eq. (A5) the solution which satisfies the normalization condition $\bar{\omega}_{\beta}(\eta=0)=1$, and with $\beta$ within the range $0 \leqslant \beta \leqslant 5 / 6$, so that the power of $\rho(\vec{r})$ in Eq. (A2) is positive, the weight function is given by

$$
\bar{\omega}_{\beta}(\eta)=2-\frac{5}{3 \beta}+\frac{1}{3 \beta} \sqrt{(5-3 \beta)^{2}+5\left[\pi_{L}^{-1}(\eta)-1-3 \eta^{2}\right]} .
$$

Requiring $\bar{\omega}_{\beta}$ to be real places a stricter limit on $\beta$ : $\beta$ $\leqslant 0.5991$. With this choice of weight function, the functional recovers the LRT limit, and in the limit of uniform density it reduces to the Thomas-Fermi functional. In the limit $\eta \rightarrow \infty$ we have

$$
\bar{\omega}_{\beta}(\eta) \rightarrow C_{1}+A / \eta^{2}+\cdots,
$$

where

$$
C_{1}=2-\frac{5}{3 \beta}+\frac{1}{3 \beta} \sqrt{17-30 \beta+9 \beta^{2}} .
$$

The constant $C_{1}$ gives rise to a Dirac delta function in the real space; therefore, it is convenient to define a "modified" weight function

$$
\tilde{\omega}_{\beta}(\eta)=\bar{\omega}_{\beta}(\eta)-C_{1},
$$

so that every convolution involving $\omega_{\beta}$, such as in Eq. (A3), becomes

$$
G(\vec{r}) * \omega_{\beta}\left(2 k_{F}^{0} r\right)=C_{1} G(\vec{r})+G(\vec{r}) * \tilde{\omega}_{\beta}\left(2 k_{F}^{0} r\right) .
$$

An important limit is when the mean electron density, and therefore $k_{F}^{0}$, vanishes as for instance in a finite system. Now the convolutions involving the "modified" weight function vanish because $\eta=q / 2 k_{F}^{0} \rightarrow \infty$ and $\tilde{\omega}(\eta)$ vanishes. Consequently, $\widetilde{k}(\vec{r})=C_{1} k(\vec{r})$, and the kinetic-energy functional becomes $T_{s}[\rho]=T_{W}[\rho]+C_{1}^{2} T_{T F}[\rho]$, and when $\beta=4 / 9, C_{1}$ $=0$.

\section{APPENDIX B: POSITION-DEPENDENT CHEMICAL POTENTIAL}

The functional derivative of Eq. (7) gives

$$
\mu(\vec{r})=\mu_{W}(\vec{r})+\mu_{\beta}(\vec{r})+V_{\mathrm{ext}}(\vec{r})+V_{H}(\vec{r})+V_{x c}[\rho(\vec{r})],
$$

where

$$
\begin{gathered}
\mu_{W}(\vec{r})=\frac{1}{8} \frac{|\vec{\nabla} \rho(\vec{r})|^{2}}{\rho(\vec{r})^{2}}-\frac{1}{4} \frac{\nabla^{2} \rho(\vec{r})}{\rho(\vec{r})}, \\
V_{H}(\vec{r})=\int d \vec{s} \frac{\rho(\vec{s})}{|\vec{r}-\vec{s}|},
\end{gathered}
$$

and, in terms of the modified weight function,

$$
\begin{aligned}
\mu_{\beta}(\vec{r})= & \frac{3}{10}\left[(5 / 3-2 \beta) \rho(\vec{r})^{2 / 3-2 \beta} \widetilde{k}(\vec{r})^{2}\right. \\
& \left.+2 \beta\left(3 \pi^{2}\right)^{1 / 3} \rho(\vec{r})^{\beta-1} h(\vec{r})\right],
\end{aligned}
$$

with

$$
h(\vec{r})=f(\vec{r}) * \tilde{\omega}_{\beta}\left(2 k_{F}^{0} r\right),
$$

where $f(\vec{r})=\widetilde{k}(\vec{r}) \rho(\vec{r})^{5 / 3-2 \beta}$. The product $\mu(\vec{r}) \psi(\vec{r})$, is the "driving force" for the dynamical minimization of the energy functional; see Eq. (15). If the various powers of the density appearing in $\mu_{\beta}(\vec{r}) \psi(\vec{r})$ are to remain positive, so that this driving force is not to diverge in regions where the density vanishes, then $1 / 2 \leqslant \beta \leqslant 7 / 12$. In practice we have found that for $\beta=0.51$ the minimization always proves possible.

\section{APPENDIX C: CONSTRUCTING THE LOCAL PSEUDOPOTENTIAL FROM AN INFINITE SYSTEM}

In an infinite system most of the previous expressions diverge, because the integrals extend to all space, and the integrands do not vanish for large distances. Moreover, the normalization constraint must be redefined, because the total number of electrons is infinite.

To avoid these problems, one has to take into account the similar divergencies which appear in the "ionic" part of the total energy. This amounts to using "difference" functions, which are obtained by subtracting from the total functions their corresponding limits for large distances, and redefining the chemical potential as the Lagrange multiplier associated with the normalization of the "displaced" density $n(\vec{r})$ $=\rho(\vec{r})-\rho_{0}$. In this way we define the following functions

(i) $\Delta_{\alpha}(\vec{r})=\rho(\vec{r})^{\alpha}-\rho_{0}^{\alpha}=\left(\rho_{0}+n(\vec{r})\right)^{\alpha}-\rho_{0}^{\alpha}$.

In particular, for $\alpha=1, \Delta_{1}(\vec{r})=n(\vec{r})$, the displaced density.

(ii) $\chi(\vec{r})=k(\vec{r})-\left(3 \pi^{2}\right)^{1 / 3} \rho_{0}^{\beta}$.

(iii) $\tilde{\chi}(\vec{r})=\widetilde{k}(\vec{r})-\left(3 \pi^{2}\right)^{1 / 3} \rho_{0}^{\beta}$.

(iv) $\phi(\vec{r})=f(\vec{r})-\left(3 \pi^{2}\right)^{1 / 3} \rho_{0}^{5 / 3-\beta}$.

(v) $\eta_{2}(\vec{r})=h(\vec{r})-\left(1-C_{1}\right)\left(3 \pi^{2}\right)^{1 / 3} \rho_{0}^{5 / 3-\beta}$.

(vi) $\tilde{\mu}_{\beta}(\vec{r})=\mu_{\beta}(\vec{r})-\frac{1}{2}\left(k_{F}^{0}\right)^{2}$.

(vii) $v_{\text {ext }}(\vec{r})=V_{\text {ext }}(\vec{r})-v_{\text {jell }}(\vec{r})$, where $v_{\text {jell }}(\vec{r})$ is the potential created by a uniform background of positive charge with density $\rho_{0}$.

(viii) $v_{x c}(\vec{r})=V_{x c}\left[\rho_{0}+n(\vec{r})\right]-V_{x c}\left[\rho_{0}\right]$.

In terms of these functions, the Euler equation now becomes

$$
\begin{aligned}
& v_{\mathrm{ext}}(\vec{r})+\int d \vec{s} n(\vec{s}) /|\vec{r}-\vec{s}|+v_{\mathrm{xc}}(\vec{r})+\frac{1}{8} \frac{|\vec{\nabla} n(\vec{r})|^{2}}{\left[\rho_{0}+n(\vec{r})\right]^{2}} \\
& -\frac{1}{4} \frac{\nabla^{2} n(\vec{r})}{\rho_{0}+n(\vec{r})}+\tilde{\mu}_{\beta}(\vec{r})-\mu^{\prime}=0
\end{aligned}
$$

with 


$$
\tilde{\mu}_{\beta}(\vec{r})=\frac{3}{10}\left[\tilde{\mu}_{A}(\vec{r})+\tilde{\mu}_{B}(\vec{r})+\tilde{\mu}_{C}(\vec{r})+\tilde{\mu}_{D}(\vec{r})\right],
$$

where

$$
\begin{gathered}
\tilde{\mu}_{A}(\vec{r})=\left(\frac{5}{3}-2 \beta\right) \rho_{0}^{2 / 3-2 \beta}\left[2\left(3 \pi^{2}\right)^{1 / 3} \rho_{0}^{\beta} \tilde{\chi}(\vec{r})+\tilde{\chi}(\vec{r})^{2}\right], \\
\tilde{\mu}_{B}(\vec{r})=\left(\frac{5}{3}-2 \beta\right) \Delta_{2 / 3-2 \beta}(\vec{r})\left[\left(3 \pi^{2}\right)^{1 / 3} \rho_{0}^{\beta}+\tilde{\chi}(\vec{r})\right]^{2}, \\
\tilde{\mu}_{C}(\vec{r})=2 \beta C_{1}\left(3 \pi^{2}\right)^{1 / 3}\left[\rho_{0}^{2 / 3-\beta} \tilde{\chi}(\vec{r})+\left(3 \pi^{2}\right)^{1 / 3} \rho_{0}^{\beta} \Delta_{2 / 3-\beta}(\vec{r})\right. \\
\left.+\Delta_{2 / 3-\beta}(\vec{r}) \tilde{\chi}(\vec{r})\right],
\end{gathered}
$$

and

$$
\begin{aligned}
\tilde{\mu}_{D}(\vec{r})= & 2 \beta\left(1-C_{1}\right)\left(3 \pi^{2}\right)^{2 / 3} \rho_{0}^{5 / 3-2 \beta} \Delta_{\beta-1}(\vec{r}) \\
& +2 \beta\left(3 \pi^{2}\right)^{1 / 3}\left[\rho_{0}+n(\vec{r})\right]^{\beta-1} \eta_{2}(\vec{r}) .
\end{aligned}
$$

In the last equation, $\eta_{2}(\vec{r})=\phi(\vec{r}) * \tilde{\omega}_{\beta}\left(2 k_{F}^{0} r\right)$ and

$$
\begin{aligned}
\phi(\vec{r})= & \rho_{0}^{5 / 3-2 \beta} \tilde{\chi}(\vec{r})+\left(3 \pi^{2}\right){ }^{1 / 3} \rho_{0}^{\beta} \Delta_{5 / 3-2 \beta}(\vec{r}) \\
& +\Delta_{5 / 3-2 \beta}(r) \tilde{\chi}(\vec{r}) .
\end{aligned}
$$

Summarizing, to evaluate $\tilde{\mu}_{\beta}(\vec{r})$ from the displaced density $n(\vec{r})$ we take the following steps:

(1) Compute $\Delta_{\alpha}(\vec{r})$ for $\alpha=5 / 3-2 \beta, 2 / 3-2 \beta, 2 / 3-\beta$, and $\beta-1$.

(2) Compute $\chi(\vec{r})$ and FT to obtain $\chi(\vec{q})$.

(3) Compute $\tilde{\chi}(\vec{q})=C_{1} \chi(\vec{q})+\chi(\vec{q}) \tilde{\omega}_{\beta}\left(q / 2 k_{F}^{0}\right)$, and by an inverse FT obtain $\tilde{\chi}(\vec{r})$.

(4) Compute $\phi(\vec{r})$ according to Eq. (C7), and FT to obtain $\phi(\vec{q})$.

(5) Compute $\eta_{2}(\vec{q})=\phi(\vec{q}) \tilde{\omega}_{\beta}\left(q / 2 k_{F}^{0}\right)$ and inverse FT to obtain $\eta_{2}(\vec{r})$.

(6) Apply Eqs. (C2)-(C6) to obtain $\tilde{\mu}_{\beta}(\vec{r})$.

When our system is one atom in a jellium vacancy, the external potential is given by

$$
v_{\text {ext }}(\vec{r})=v_{\mathrm{ps}}(r)+v_{\text {cav }}(r),
$$

i.e., the sum of the potential created by the cavity and the ionic pseudopotential. Substituting into Eq. (C1), we directly obtain $v_{\mathrm{ps}}(r)$ once we compute all the other terms, which are calculated from $n(r)$. Note that all the functions have spherical symmetry, which leads to simple expressions for the gradient, the Laplacian, and also the Fourier tranforms.
*Email address: david@liq1.fam.cie.uva.es

${ }^{1}$ P. Hohenberg and W. Kohn, Phys. Rev. 136, 864 (1964).

${ }^{2}$ W. Kohn and L.J. Sham, Phys. Rev. 140, A1133 (1965).

${ }^{3}$ W.H. Young, Rep. Prog. Phys. 55, 1769 (1992).

${ }^{4}$ D.J. González, D.A. Ng, and M. Silbert, J. Non-Cryst. Solids 117/118, 469 (1990).

${ }^{5}$ S. Kambayashi and G. Kahl, Phys. Rev. A 46, 3255 (1992).

${ }^{6}$ M. Canales, L.E. González, and J.A. Padró, Phys. Rev. E 50, 3656 (1994).

${ }^{7}$ I. Ebbsjo, T. Kinell, and J. Waller, J. Phys. C 13, 1865 (1980).

${ }^{8}$ L. Dagens, M. Rasolt, and R. Taylor, Phys. Rev. B 11, 2726 (1975).

${ }^{9}$ G. Jacucci, R. Taylor, A. Tenenbaum, and N. van Doan, J. Phys. F: Met. Phys. 11, 783 (1981).

${ }^{10}$ J.L. Bretonnet and C. Regnaut, Phys. Rev. B 31, 5071 (1985).

${ }^{11}$ J. Hafner and W. Jank, Phys. Rev. B 42, 11530 (1990).

${ }^{12}$ W. A. Harrison, Pseudopotentials in the Theory of Metals (Benjamin, New York, 1966).

${ }^{13}$ N.W. Ashcroft, Phys. Lett. 23, 48 (1966).

${ }^{14}$ J. Chihara and S. Kambayasi, J. Phys.: Condens. Matter 6, 10221 (1994).

${ }^{15}$ J.A. Anta and A.A. Louis, Phys. Rev. B 61, 11400 (2000).

${ }^{16}$ F. Shimojo, Y. Zempo, K. Hoshino, and M. Watabe, Phys. Rev. B 52, 9320 (1995).

${ }^{17}$ F. Shimojo, Y. Zempo, K. Hoshino, and M. Watabe, J. Non-Cryst. Solids 205-207, 983 (1996).

${ }^{18}$ B.J.C. Cabral and J.L. Martins, Phys. Rev. B 51, 872 (1995).

${ }^{19}$ G. Kresse, J. Non-Cryst. Solids 205-207, 833 (1996); G. Kresse and J. Hafner, Phys. Rev. B 55, 7539 (1997).

${ }^{20}$ S.R. Bickham, O. Pfaffenzeller, L.A. Collins, J.D. Kress, and
D. Hohl, Phys. Rev. B 58, R11 813 (1998).

${ }^{21}$ M. Pearson, E. Smargiassi, and P.A. Madden, J. Phys.: Condens. Matter 5, 3221 (1993).

${ }^{22}$ See, for instance, M.I. Aoki and K. Tsumuraya, Phys. Rev. B 56, 2962 (1997).

${ }^{23}$ See, for instance, A. Vichare and D.G. Kanhere, J. Phys.: Condens. Matter 10, 3309 (1998); A. Aguado, J.M. López, J.A. Alonso, and M.J. Stott, J. Chem. Phys. 111, 6026 (1999).

${ }^{24}$ M. Foley, E. Smargiassi, and P.A. Madden, J. Phys.: Condens. Matter 6, 5231 (1994); J.A. Anta and P.A. Madden, ibid. 11, 6099 (1999).

${ }^{25}$ D.J. González, L.E. González, J.M. López, and M.J. Stott, J. Chem. Phys. 115, 2373 (2001).

${ }^{26}$ J.A. Anta, B.J. Jesson, and P.A. Madden, Phys. Rev. B 58, 6124 (1998).

${ }^{27}$ S. Gómez, L.E. González, D.J. González, M.J. Stott, S. Dalgiç, and M. Silbert, J. Non-Cryst. Solids 250-252, 163 (1999).

${ }^{28}$ R. Winter, F. Hensel, T. Bodensteiner, and W. Gläser, Ber. Bunsenges. Phys. Chem. 91, 1327 (1987).

${ }^{29}$ D Jovic, I Padureanu, and S. Rapeanu, in Liquid Metals 1976, edited by R. Evans and D.A. Greenwood, IOP Conf. Proc. No. 30 (Institute of Physics, Bristol, 1977), p. 120.

${ }^{30}$ S. Takeda, S. Harada, S. Tamaki, and Y. Waseda, J. Phys. Soc. Jpn. 60, 2241 (1991).

${ }^{31}$ Y. Waseda, The Structure of Non-Crystalline Materials (McGrawHill, New York, 1980).

${ }^{32}$ IAMP database of SCM-LIQ, Tohoku University. URL: http:// www.iamp.tohoku.ac.jp/database/scm.

${ }^{33}$ T. Scopigno, U. Balucani, G. Ruocco, and F. Sette, Phys. Rev. E 63, 011210 (2000). 
${ }^{34}$ See, for instance, M.P. Allen, and D.J. Tildesley, Computer Simulation of Liquids (Oxford, University Press, Oxford, 1987).

${ }^{35}$ E.S. Kryachko and E.V. Ludeña, Energy Density Functional Theory of Many-Electron Systems (Kluwer, London, 1990), and references therein.

${ }^{36}$ F. Perrot, J. Phys.: Condens. Matter 6, 431 (1994).

${ }^{37}$ E. Smargiassi and P.A. Madden, Phys. Rev. B 49, 5220 (1994).

${ }^{38}$ M. Foley and P.A. Madden, Phys. Rev. B 53, 10589 (1996).

${ }^{39}$ Y.A. Wang, N. Govind, and E.A. Carter, Phys. Rev. B 58, 13465 (1998); 60, 16350 (1999).

${ }^{40}$ E. Chacón, J.E. Alvarellos, and P. Tarazona, Phys. Rev. B 32, 7868 (1985).

${ }^{41}$ P. Garcia-González, J.E. Alvarellos, and E. Chacón, Phys. Rev. A 54, 1897 (1996); Phys. Rev. B 53, 9509 (1996); 57, 4857 (1998).

${ }^{42}$ See, for instance, N. Trouiller and J.L. Martins, Phys. Rev. B 43, 1993 (1991).

${ }^{43}$ L.E. González, A. Meyer, M.P. Iñiguez, D.J. González, and M. Silbert, Phys. Rev. E 47, 4120 (1993).

${ }^{44}$ H.J. Seemann and F.K. Klein, Z. Angew. Math. Phys. 19, 368 (1965).

${ }^{45}$ S. Watson, B.J. Jesson, E.A. Carter, and P.A. Madden, Europhys. Lett. 41, 37 (1998).

${ }^{46}$ U. Balucani and M. Zoppi, Dynamics of the Liquid State (Clarendon, Oxford 1994).

${ }^{47}$ U. Balucani, A. Torcini, and R. Vallauri, Phys. Rev. A 46, 2159 (1992); Phys. Rev. B 47, 3011 (1993).

${ }^{48}$ J. Casas, D.J. González, and L.E. González, Phys. Rev. B 60, 10094 (1999).

${ }^{49}$ M.M.G. Alemany, J. Casas, C. Rey, L.E. González, and L.J. Gallego, Phys. Rev. E 56, 6818 (1997).

${ }^{50}$ P.E. Blochl and M. Parrinello, Phys. Rev. B 45, 9413 (1992).

${ }^{51}$ D.R. Hamann, M. Schluter, and C. Chiang, Phys. Rev. Lett. 43, 1494 (1979).

${ }^{52}$ G.B. Bachelet, D.R. Hamann, and M. Schluter, Phys. Rev. B 26, 4199 (1982).
${ }^{53}$ D.R. Hamann, Phys. Rev. B 40, 2980 (1989).

${ }^{54}$ D. Alfe and M.J. Gillan, Phys. Rev. Lett. 81, 5161 (1998).

${ }^{55}$ C. Morkel and W. Glaser, Phys. Rev. A 33, 3383 (1986); C. Morkel, C. Gronemeyer, W. Glaser, and J. Bosse, Phys. Rev. Lett. 58, 1873 (1987).

${ }^{56}$ G. Wahnström and L. Sjögren, J. Phys. C 15, 401 (1982).

${ }^{57}$ P. Verkerk, J.H. Builtjes, and I.M. de Schepper, Phys. Rev. A 31, 1731 (1985).

${ }^{58}$ P. Verkerk, J. Westerweel, U. Bafile, and I. M. de Schepper, in Static and Dynamic Properties of Liquids edited by M. Davidovic and A.K. Soper (Springer, Heidelberg, 1989).

${ }^{59}$ W. Montfrooy, I. de Schepper, J. Bosse, W. Glaser, and C. Morkel, Phys. Rev. A 33, 1405 (1986).

${ }^{60}$ A. Torcini, U. Balucani, P.H.K. de Jong, and P. Verkerk, Phys. Rev. E 51, 3126 (1995).

${ }^{61}$ D.L. Price, K.S. Singwi, and M.P. Tosi, Phys. Rev. B 2, 2983 (1970).

${ }^{62}$ F. Shimojo, K. Hoshino, and M. Watabe, J. Phys. Soc. Jpn. 63, 141 (1994).

${ }^{63}$ S. Kambayashi and G. Kahl, Phys. Rev. A 46, 3255 (1992).

${ }^{64}$ J. Casas, D.J. González, L.E. González, M.M.G. Alemany, and L.J. Gallego, Phys. Rev. B 62, 12095 (2000).

${ }^{65}$ G. M. B. Webber and R. W. B Stephens, in Physical Acoustics, edited by W.P. Mason (Academic Press, New York, 1968), Vol. IVB.

${ }^{66}$ H. Sinn and E. Burkel, J. Phys.: Condens. Matter 8, 9369 (1996); H. Sinn, F. Sette, U. Bergmann, Ch. Halcoussis, M. Krisch, R. Verbeni, and E. Burkel, Phys. Rev. Lett. 78, 1715 (1997).

${ }^{67}$ C. Pilgrim, S. Hosokawa, H. Saggau, H. Sinn, and E. Burkel, J. Non-Cryst. Solids 250-252, 96 (1999).

${ }^{68}$ B.J. Palmer, Phys. Rev. E 49, 359 (1994).

${ }^{69}$ U. Balucani, J.P. Brodholt, P. Jedlovszky, and R. Vallauri, Phys. Rev. E 62, 2971 (2000).

${ }^{70}$ M. Shimoji and T. Itami, Atomic Transport in Liquid Metals, (Trans. Tech, Aedermannsdorf, 1986). 